\title{
Calculation of the entropy of binary hard sphere mixtures from pair correlation functions
}

\author{
Brian B. Laird and A. D. J. Haymet \\ Department of Physical and Theoretical Chemistry, University of Sydney, NSW 2006 Australia
}

(Received 28 April 1992; accepted 20 May 1992)

The evaluation of the entropy of a liquid has wide applications in protein folding, ${ }^{1}$ micelle formation, ${ }^{2}$ membrane studies, ${ }^{2}$ the hydrophobic effect, ${ }^{3}$ ion solvation, ${ }^{4}$ and other studies of solutions, ${ }^{5-9}$ but remains a difficult problem, both for computational and analytic statistical mechanics. In this paper we evaluate the nonideal contribution to the entropy of mixing for a simple binary liquid, using an extension of simple, closed-form equations for single-component liquids.

In a recent paper, ${ }^{10}$ we investigated three approximate expressions for the total entropy of a single-component fluid in terms of the pair correlation function, $g(r)$. For the six liquids studied, the calculated entropy was within $10 \%$ of the simulation value over almost the entire fluid density range. In light of this success, we examine in this communication two of these approximations for the binary hardsphere mixture. We show that the approximation for the entropy obtained from the incompressible limit remains accurate and useful.

For a single-component system in the canonical ensemble, an expansion for the entropy in terms of multiparticle correlation functions was derived by Green. ${ }^{11}$ Later, Nettleton and Green ${ }^{12}$ and Raveché ${ }^{13}$ determined the corresponding expression for the grand canonical ensemble. That the two expressions differ ensures that they yield the same results in the thermodynamic limit (that is, in spite of the difference in asymptotic behavior of the mutliparticle correlation functions between the two ensembles). In the grand canonical ensemble, the correlations approach unity at large particle separation, but those of the canonical ensemble approach a value that differs from unity by a factor proportional to the isothermal compressibility and inversely proportional to the number of particles. ${ }^{14}$ Through a clever rearrangement of terms, Baranyai and Evans ${ }^{15}$ showed that the grand canonical expression is, in fact, an ensemble invariant one, namely, it yields the same result whether canonical or grand canonical correlation functions are used.

The generalization of the grand canonical (and thus ensemble invariant) entropy expansion to mixtures was obtained by Hernando. ${ }^{16}$ For a multicomponent system at temperature $T$, occupying a volume $V$, with an average total number of particles $N$, the entropy may be written

$$
\begin{aligned}
& \frac{S}{N k}=s^{(0)}+s^{(2)}+s^{(3)}+\cdots, \\
& s^{(0)}=1+\sum_{\alpha} x_{\alpha}\left[\frac{y_{\alpha}}{2}-\ln \left(\rho_{\alpha} \Lambda_{\alpha}^{3}\right)\right],
\end{aligned}
$$

$$
\begin{aligned}
s^{(2)}= & -\frac{\rho}{2} \sum_{\alpha \beta} x_{\alpha} x_{\beta} \int_{V} d \mathbf{r}\left[g_{\alpha \beta}^{(2)}(\mathbf{r}) \ln g_{\alpha \beta}^{(2)}(\mathbf{r})-g_{\alpha \beta}^{(2)}(\mathbf{r})+1\right] \\
s^{(3)}= & -\frac{\rho^{2}}{6 V} \sum_{\alpha, \beta, \gamma} x_{\alpha} x_{\beta} x_{\gamma} \int_{V} \int_{V} \int_{V} d \mathbf{r}_{\alpha} d \mathbf{r}_{\beta} d \mathbf{r}_{\gamma} \\
& \times\left[g_{\alpha \beta \gamma}^{(3)}\left(\mathbf{r}_{\alpha \beta}, \mathbf{r}_{\beta \gamma}, \mathbf{r}_{\alpha \gamma}\right) \ln \left(\frac{g_{\alpha \beta \gamma}^{(3)}\left(\mathbf{r}_{\alpha, \beta}, \mathbf{r}_{\beta \gamma}, \mathbf{r}_{\alpha \gamma}\right)}{g_{\alpha \beta}^{(2)}\left(\mathbf{r}_{\alpha \beta}\right) g_{\beta \gamma}^{(2)}\left(\mathbf{r}_{\beta \gamma}\right) g_{\alpha \gamma}^{(2)}\left(\mathbf{r}_{\alpha \gamma}\right)}\right)\right. \\
& -g_{\alpha \beta \gamma}^{(3)}\left(\mathbf{r}_{\alpha \beta}, \mathbf{r}_{\beta \gamma} \mathbf{r}_{\alpha \gamma}\right) \\
& \left.+3 g_{\alpha \beta}^{(2)}\left(\mathbf{r}_{\alpha \beta}\right) g_{\beta \gamma}^{(2)}\left(\mathbf{r}_{\beta \gamma}\right)-3 g_{\alpha \beta}^{(2)}\left(\mathbf{r}_{\alpha \beta}\right)+1\right]
\end{aligned}
$$

where $k$ is the Boltzmann constant, $\alpha, \beta$, and $\gamma$ index particle species, the number density $\rho=N / V$, and $x_{\alpha}, v_{\alpha}$, and $\Lambda_{\alpha}$ are the mole fraction, number of degrees of freedom, and thermal wavelength of component $\alpha$, respectively.

In liquid phase statistical mechanics, usually only the pair correlation functions are known. To be of practical use, the entropy expression [Eqs. (1)-(5)] is usually truncated after the second-order term, yielding the following approximate expression for the reduced excess entropy per particle

$$
\begin{aligned}
& s \equiv \frac{S-S_{\text {ideal }}}{N k} \approx s^{(2)} \\
& s^{(2)}=-\frac{\rho}{2} \sum_{\alpha, \beta} x_{\alpha} x_{\beta} \int_{V} d \mathbf{r}\left[g_{\alpha \beta}^{(2)}(\mathbf{r}) \ln g_{\alpha \beta}^{(2)}(\mathbf{r})-g_{\alpha \beta}^{(2)}(\mathbf{r})+1\right] .
\end{aligned}
$$

Note that the ideal entropy which has been subtracted in Eq. (5) is for an ideal mixture and hence includes the ideal entropy of mixing.

The ensemble invariant expression for the entropy of a fluid mixture [(Eqs. (1)-(4)] contains terms of two types. The first type involves integrals over expressions of the form $g^{(n)} \ln (\cdots)$. Hernando ${ }^{16}$ has shown that these terms vanish for $n \geqslant 3$ within the generalized superposition approximation (GSA) ${ }^{17}$ Since this approximation is accurate for low to medium densities, any error in the entropy calculated using $s^{(2)}$ in this density region must result from the omission of the remainder terms for $n \geqslant 3$.

For densities high enough that the fluid is relatively incompressible, it is possible to approximate the remainder terms in a very simple way. In the limit that density fluctuations are small (that is, the compressibility is small), 
the difference between the grand canonical correlation functions and those of the canonical ensemble approaches zero. This correspondence between the two ensembles in the "incompressible limit" has been discussed extensively by Wallace. ${ }^{18}$ Since the sum of all the higher-order remainder terms [those not of the form $g^{(n)} \ln (\cdots)$ ] for $n \geqslant 3$ is exactly $-\frac{1}{2}$ within the canonical ensemble, ${ }^{15}$ the grand canonical entropy should approach

$$
s \approx s^{\text {inc }} \equiv s^{(2)}-\frac{1}{2}
$$

in the incompressible limit, provided the density is still low enough that the GSA is valid. The above "incompressible limit" expression was derived for the single-component case, but holds for a multicomponent fluid as well, because the sum of all the remainder terms evaluated using canonical correlation functions must exactly compensate for the difference between the ideal $\left(s^{(0)}\right)$ parts of the grand canonical and canonical entropy expansions, ${ }^{14}$ which is the same for both single- and multicomponent fluids.

We now evaluate the above two approximations for the entropy, $s^{(2)}$ and $s^{\text {inc }}$, for mixtures of hard spheres, at various densities and compositions. The results are compared with a relatively accurate empirical fit to the known exact entropy, and hence the region of validity of the two approximations is mapped out.

A mixture of hard spheres with additive diameters is defined by the following pairwise-additive potential energy, between a particle of type $\alpha$ with diameter $\sigma_{\alpha}$ and a particle of type $\beta$ with diameter $\sigma_{\beta}$

$$
u_{\alpha \beta}(r)=\left\{\begin{array}{ll}
\infty, & r / \sigma_{\alpha \beta}<1 \\
0, & r / \sigma_{\alpha \beta}>1
\end{array},\right.
$$

where $\sigma_{\alpha \beta}=\left(\sigma_{\alpha}+\sigma_{\beta}\right) / 2$. Such a potential is a useful first choice to test the various entropy expressions for mixtures. The excess Helmholtz free energy is purely entropic, and furthermore good, approximate, closed form expressions exist for the structural and thermodynamic properties, greatly simplifying the numerical evaluations.

Another advantage of the hard-sphere mixture is that for a fluid with fixed diameters and mole fractions, the excess entropy is a function of the total density alone: The temperature plays no role because there is no explicit energy scale. Instead of using the total density, the results will be presented below as a function of the total packing fraction

$$
\eta=\frac{\pi \rho}{6} \sum_{\alpha} x_{\alpha} \sigma_{\alpha}^{3}
$$

which, for a binary mixture with diameter ratio $\sigma_{1} / \sigma_{2}=d$, and mole fractions $x_{1}$ and $x_{2}=\left(1-x_{1}\right)$, may be written

$$
\eta=\frac{\pi \rho \sigma_{2}^{3}}{6}\left[x_{2}+\left(1-x_{2}\right) d^{3}\right]
$$

(In what follows we assume without loss of generality that $\sigma_{2}>\sigma_{1}$, namely, $0 \leqslant d \leqslant 1$.) This packing fraction is the fraction of the total volume occupied by the spheres.

To evaluate $s^{(2)}$ for a specific hard-sphere mixture, it is necessary to have a good approximation to the pair corre-

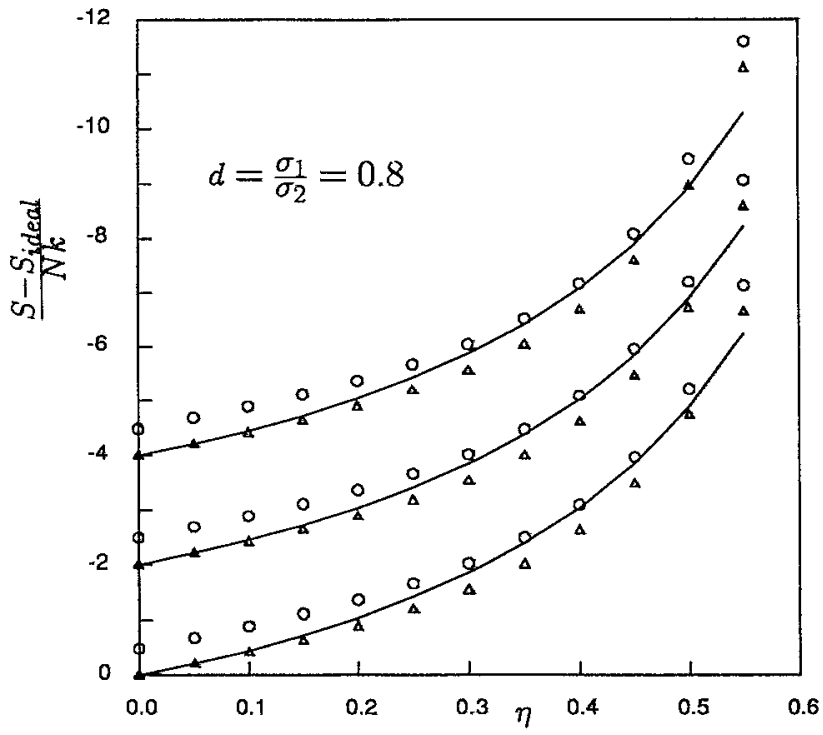

FIG. 1. Excess entropy for a binary hard-sphere fluid with diameter ratio, $d=0.8$ for large-sphere mole fractions $x_{2}=0.2$ (lower curves), 0.5 (middle curves), and 0.8 (upper curves) - the middle and upper sets of curves are shifted by 2 and 4 , respectively. The circles give $s^{(2)}$, the triangles show the incompressible limit, $s^{(2)}-1 / 2$ and the solid lines show the modified Mansoori fit (Ref. 22).

lation functions, $g_{\alpha \beta}(r)$. For these functions, we use the Percus-Yevick approximation. ${ }^{19}$ For an additive hard sphere mixture, a closed-form analytic solution to this approximation was obtained by Lebowitz. ${ }^{20}$ This approximation is generally very good for hard-core potentials at densities not too close to freezing.

Our results will be tested below against an accurate equation of state, which yields the entropy determined by computer simulations. We use a modified version of the Mansoori ${ }^{21}$ equation of state due to Kranendonk and Frenkel. $^{22}$ This function accurately reproduces the simulation data over most of the fluid parameter space.

We have calculated both $s^{(2)}$ and $s^{\text {inc }}=s^{(2)}-\frac{1}{2}$ as a function of the total packing fraction, $\eta$ for a variety of diameter ratios and mole fractions. These quantities, together with the "exact" value from the modified Mansoori fit, are plotted in Fig. 1 for a diameter ratio, $d=\sigma_{1} / \sigma_{2}=0.8$ and three different large sphere mole fractions: $x_{2}=0.2$ (lower curve), $x_{2}=0.5$ (middle curve), and $x_{2}=0.8$ (upper curve). The three curves are very similar. This results because, for diameter ratios not too different from unity (nearly equal sized spheres), the entropy as a function of total packing fraction is very nearly independent of the concentration ratios. As for single-component liquids ${ }^{10}$ the incompressible limit approximation is very good for intermediate packing fractions between about 0.3 and 0.45 , but poor at low densities where the fluid is quite compressible. At low packing fractions (below about 0.2 ) the simple second-order truncation $s^{(2)}$ yields a fine approximation to the "exact" entropy. Interestingly, $s^{(2)}$ again becomes very nearly exact just above a packing fraction of 0.5 . This phenomena is also observed for single-component fluids, and it has been shown to result from a fortuitous cancellation of 


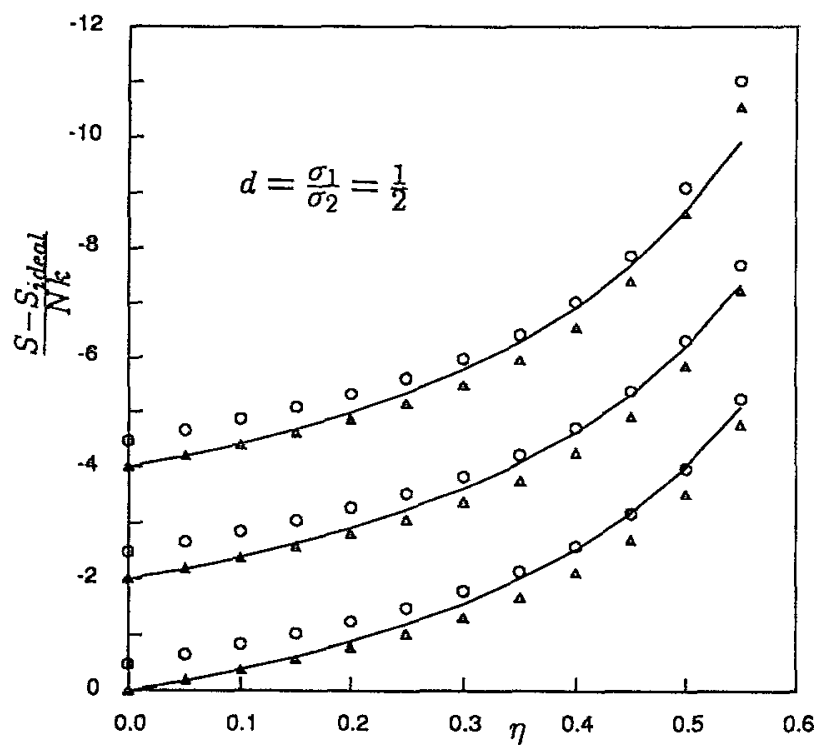

FIG. 2. Same as Fig. 1, except for diameter ratio, $d=1 / 2$.

the higher-order $g^{(n)} \ln (\cdots)$ terms $(n>3) .{ }^{23}$

As the diameter ratio decreases (that is, as the sphere diameters becomes more asymmetric), the entropy as a function of the total packing fraction becomes more strongly dependent upon the mole fraction. In Figs. 2 and 3 , the two approximations and the simulation result are plotted for diameter ratios $d=1 / 2$ and $1 / 3$, respectively. At these two diameter ratios, the behavior is similar to $d=0.8$. One obvious difference is that for the more asymmetric cases, the region of validity of $s^{(2)}$ at low packing fraction increases as the fraction of the small spheres increases. In addition, the region of validity of the "incompressible limit" shifts to slightly higher packing fractions. These phenomena become more pronounced as the asym-

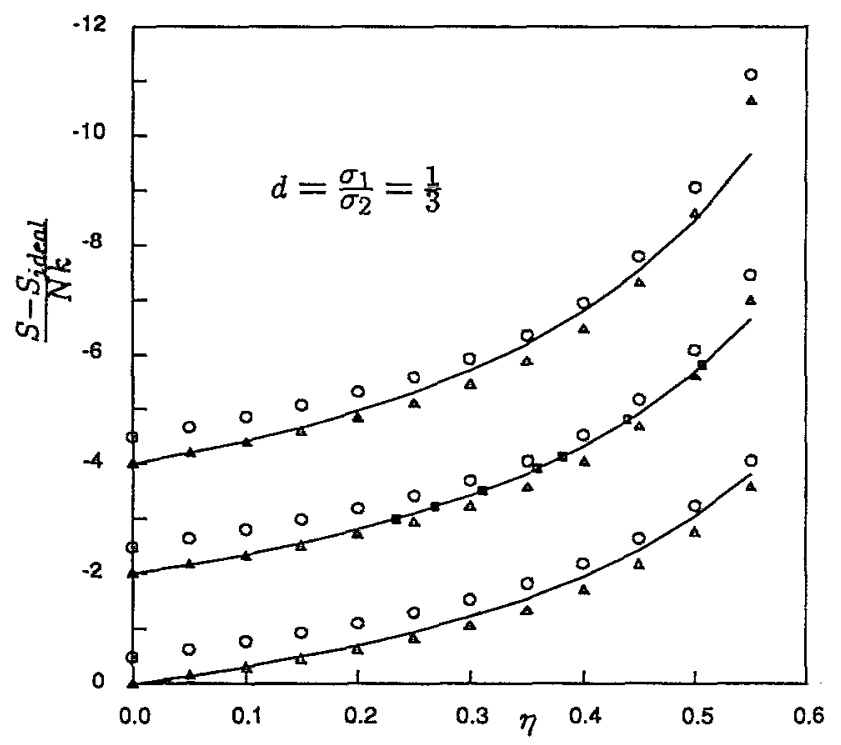

FIG. 3. Same as Fig. 1, except for diameter ratio, $d=1 / 3$. The squares on the middle curve are the results of Monte Carlo simulation (Ref. 21). metry becomes larger. Based on the results of computer simulation of phase coexistence in hard-sphere mixtures, ${ }^{24}$ these trends are likely to be correlated with an increase in the liquid packing fraction at freezing, $\eta_{f}$ as the fraction of smaller spheres becomes larger. Additional information can be gained from plotting ${ }^{25}$ the entropy of the various mixtures as a function of the ratio $\eta / \eta_{f}$.

The summary, the qualitative behavior and quantitative accuracy of the entropy expansions for mixtures of hard spheres has been shown to be similar to those of the single component case. In particular, the incompressible limit is a valuable, approximate expression for the entropy of dense liquids. Although further study is warranted, it is clear that these results give support to the use of such methods in the analysis of more complicated systems, such as aqueous solutions, ${ }^{26}$ where entropic effects play an important role, but for which thermodynamic data is difficult to obtain.

This research was supported in part by the U.S. National Science Foundation through Grant No. CHE8913006, and in part by the Australian Research Council (ARC) (Grant No. A29131271). A grant of computer time from the Utah Supercomputing Institute (USI), funded by Utah and IBM Corporation, is acknowledged gratefully. We also thank Dr. Douglas Henderson for the use of his computer program to evaluate the pair correlation functions for the binary hard-sphere mixture.

${ }^{1}$ K. A. Dill, Biochemistry 29, 7133 (1990).

${ }^{2} \mathrm{C}$. Tanford, The Hydrophobic Effect: Formation of Micelles and Biological Membranes (Wiley, New York, 1980).

${ }^{3}$ A. Ben-Naim, Hydrophobic Interactions (Plenum, New York, 1980).

${ }^{4}$ Y. Marcus, Ion Solvation (Wiley, Chichester, 1985).

${ }^{5}$ W. Kauzmann, Adv. Protein Chem. 14, 1 (1959).

${ }^{6} \mathrm{D}$. W. Davidson, Water, a comprehensive treatise, edited by F. Franks (Plenum, New York, 1973), Vol. 2, Chap. 3.

${ }^{7}$ F. Franks, Water, a comprehensive treatise edited by F. Franks (Plenum, New York, 1975), Vol. 4, Chap. 1.

${ }^{8}$ D. Y. C. Chan, D. J. Mitchell, B. W. Ninham, and B. A. Pailthorpe, Water, a comprehensive treatise, edited by F. Franks (Plenum, New York, 1979), Vol. 6, Chap. 5.

${ }^{9}$ G. Némethy, W. J. Peer, and H. A. Scheraga, Ann. Rev. Biophys. Bioeng. 10, 59 (1981).

${ }^{10}$ B. B. Laird and A. D. J. Haymet, Phys. Rev. A 45, 5680 (1992).

${ }^{11}$ H. S. Green, The Molecular Theory of Fluids (North-Holland, Amsterdam, 1952).

${ }^{12}$ R. E. Nettleton and H. S. Green, J. Chem. Phys. 29, 1365 (1958).

${ }^{13}$ H. J. Raveché, J. Chem. Phys. 55, 2242 (1971).

${ }^{14}$ J. L. Lebowitz and J. K. Percus, Phys. Rev. A 122, 1967 (1961).

${ }^{15}$ A. Baranyai and D. J. Evans, Phys. Rev. A 40, 3817 (1989).

${ }^{16}$ J. A. Hernando, Mol. Phys. 69, 319 (1990).

${ }^{17}$ I. Z. Fisher and B. L. Kopeliovich, Soviet Phys. Doklady 5, 761 (1961).

${ }^{18}$ D. C. Wallace, J. Chem. Phys. 87, 4843 (1987).

${ }^{19}$ J, K. Percus and G. J. Yevick, Phys. Rev, 110, 1 (1958).

${ }^{20}$ J. L. Lebowitz, Phys. Rev. A 133, 895 (1964).

${ }^{21}$ G. A. Mansoori, K. E. Starling, N. F. Carnahan, and T. W. Leland, J. Chem. Phys. 54, 1523 (1971).

${ }^{22}$ W. G. T. Kranendonk and D. Frenkel, Mol. Phys. 72, 715 (1991).

${ }^{23}$ A. Baranyai and D. J. Evans, Z. Narturforsch. 46a, 27 (1990).

${ }^{24}$ W. G. T. Kranendonk and D. Frenkel, Mol. Phys. 72, 679 (1991).

${ }^{25}$ B. B. Laird and A. D. J. Haymet, J. Phys. Chem., in preparation (1992).

${ }^{26}$ T. Lazaridis and M. E. Paulaitis, J. Phys. Chem. 96, 3847 (1992). 